\title{
Sincronía reproductiva interpoblacional de Agave cocui (Agavaceae) en Venezuela
}

\author{
Carmen J. Figueredo ${ }^{1}$, José Luis Villegas ${ }^{2} \&$ Jafet M. Nassar $^{1}$ \\ 1. Centro de Ecología, Instituto Venezolano de Investigaciones Científicas, A.P. 20632, Caracas 1020-A, Venezuela; \\ figueredocj@gmail.com,jafet.nassar@gmail.com \\ 2. Centro de Física, Instituto Venezolano de Investigaciones Científicas, A.P. 20632, Caracas 1020-A, Venezuela; \\ josevillega@gmail.com
}

Recibido 03-IX-2010. C Corregido 08-II-2011. Aceptado 01-III-2011.

\begin{abstract}
Interpopulation reproductive synchrony of Agave cocui (Agavaceae) in Venezuela. Agave cocui (Agavaceae) is a species with broad distribution in arid and semiarid areas of Venezuela and Colombia. Despite of its ecological importance as a source of food for wildlife, and its economic value for production of a spirit drink, studies on the reproductive ecology of the species are relatively rare. In this study, we conducted a oneyear evaluation of the flowering and fruiting phenology of A. cocui in the eight representative localities of the species' distribution in Venezuela. Within each study site, we chose an area with a minimum of 50 reproductive individuals and followed their reproductive phenophases with the help of binoculars, using six qualitative cathegories (emerging reproductive stalk, flowers, inmature fruits, mature fruits, bulbils and dry stalk) every two months. Emergence of the reproductive stalk in most of the examined populations began in September (rainy season), although this event delayed two months in a few populations. We detected significant negative correlations between precipitation and the percentage of flowering occurrence in four of the eight populations. Floral resources are available for flower visitors during approximately five months of the year (January-May). In most populations production of flowers initiated in January (dry season), and for Western Venezuela and Andean regions, the flowering main peak occurred in January. Localities from the Central and Eastern Coast exhibited the flowering peak in March, showing a delay of approximately two months with respect to other populations. Beginning of fruit set varied among localities from January to May; however, peak production of mature fruits concentrated in May, and fruit occurrence varied broadly between 5.2 and $85 \%$. Bulbil production was detected in all populations and varied greatly among them (maximum percentage per population: 26.19-92.10\%). High flowering synchronicity (Phenophase Overlapping Index: 0.756 and 0.999 ) was observed among all populations monitored in Western Venezuela, including the Andean localities. This condition might facilitate the existence of a nectar corridor from the Western Coast and nearby islands, to the Andean arid patches, which could be potentially used by nectar-feeding bats and birds dependent on agave flowers during part of the year. Rev. Biol. Trop. 59 (3): 1359-1370. Epub 2011 September 01.
\end{abstract}

Key words: Agavaceae, arid zone, flowering, nectar corridors, reproductive synchrony.

Para ecosistemas secos, se ha referido que los patrones fenológicos foliares y reproductivos de la vegetación están íntimamente relacionados con la disponibilidad de agua (Fresnillo-Fedorenko et al. 1996, Eamus \& Prior 2001, Pavón \& Briones 2001, Borchert et al. 2004), la temperatura y el fotoperíodo (Rivera et al. 2002, Borchert et al. 2004, Elliot et al. 2006). En adición a estos factores físicos, algunos autores proponen una asociación entre la fenología foliar y reproductiva de las plantas y algunas interacciones bióticas, como la herbivoría, la polinización y la dispersión de semillas (Fitter \& Fitter 2002). Por otro lado, existen evidencias que apoyan la relación entre la fenología de las plantas y los eventos en el ciclo de vida de los animales que interactúan con ellas, como son los movimientos 
estacionales de agentes polinizadores y dispersores (Janzen 1967, Mosquin 1971, Waser 1979, Waser \& Real 1979, Noma \& Yumoto 1997, Zeng et al. 2010). Con base en esta última relación, se han definido los "corredores de néctar" o "corredores de recursos", que consisten en extensiones espaciales a lo largo de las cuales especies de plantas florecen y fructifican en sincronía con la llegada de sus agentes polinizadores y dispersores (Fleming et al. 1993, Brusca et al. 2009).

A lo largo de las zonas desérticas de la costa Oeste de México y el Suroeste de Estados Unidos, las poblaciones de murciélagos antófilos de los géneros Leptonycteris Lydekker y Choeronycteris Tschudi realizan migraciones anuales (Brusca et al. 2009). Estos murciélagos se alimentan en buena proporción del néctar de las flores de agaváceas y cactáceas, cuyos períodos de floración parecen formar latitudinalmente un amplio corredor de néctar que mantiene a las poblaciones de murciélagos durante su desplazamiento (Fleming et al. 1993, Ceballos et al. 1997). Para Venezuela se ha propuesto la existencia de un patrón similar. El murciélago de los cardones, Leptonycteris curasoae Miller, es una especie restringida a las regiones áridas y semiáridas del norte de Sur América y es dependiente en un $98 \%$ de agaves y cactus columnares para su alimentación (Nassar et al. 2003). Este quiróptero realiza migraciones locales anuales, desplazándose entre los ambientes áridos de la región Norte Costera y los bolsones xéricos andinos de Venezuela y Colombia, hecho que ha sido evidenciado sólo de manera indirecta (Soriano et al. 2000, Newton et al. 2003). Por tanto, es posible que las cactáeas columnares junto con Agave cocui Trelease 1913, la única especie de agave ampliamente distribuida en Venezuela (García-Mendoza 2004), formen un corredor de néctar disponible para esta especie de murciélago durante sus movimientos anuales y posiblemente para otras especies de vertebrados voladores que hagan uso de estas plantas. La existencia de este corredor de recursos florales, además, tendría implicaciones muy importantes para la ecología reproductiva y la evolución de A. cocui y de otras plantas de zonas áridas que establezcan interacciones mutualistas de polinización y dispersión con aves y murciélagos que transiten por dicho corredor. Agentes polinizadores de larga distancia, como L. curasoae, podrían estar contribuyendo a mantener flujo genético vía polen entre las poblaciones de agave dentro del corredor, lo que promueve así la cohesión genética de esta planta suculenta en buena parte de su distribución geográfica. Para que este intercambio genético interpoblacional se pueda dar, las poblaciones de A. cocui a lo largo del eje árido Norte-Sur tendrían que presentar elevada sincronía de floración, lo que aun no ha sido demostrado.

Para A. cocui, sólo se ha realizado un estudio de fenología reproductiva en dos poblaciones del Estado Falcón (Lemus 2003). De acuerdo con este trabajo, se encontró que el crecimiento del escapo floral en A. cocui se inició a finales de la estación lluviosa y comienzos de la estación de sequía, durante los meses de enero y febrero, con máximos de floración en la época de sequía, entre marzo y abril para ambas poblaciones. Otras plantas asociadas a zonas áridas y semiáridas en Venezuela presentan un grado de solapamiento reproductivo elevado a pesar de estar geográficamente distanciadas. Por ejemplo, las cactáceas columnares quiropterófilas Stenocereus griseus (Haw.) Buxb. y Cereus repandus (L.) Mill. presentan sincronía reproductiva entre poblaciones de la Península de Paraguaná, Estado Falcón, y poblaciones en el Parque Nacional Cerro Saroche, en el Estado Lara (Nassar \& Emaldi 2008). Hasta el presente, no existen estudios en los que se haya evaluado la fenología reproductiva de $A$. cocui en todo su ámbito de distribución geográfica en Venezuela, de manera que se puedan identificar los posibles desfases temporales entre los procesos de floración de las distintas poblaciones. Es por ello que el objetivo de este estudio fue describir la fenología reproductiva de $A$. cocui en un conjunto de localidades representativas de la distribución geográfica de esta especie en Venezuela y evaluar el grado de sincronía reproductiva entre dichas localidades. 


\section{MATERIALES Y MÉTODOS}

Área de estudio: El estudio se llevó a cabo en ocho localidades representativas del área de distribución de Agave cocui en Venezuela, que fueron clasificadas en cuatro regiones (Cuadro 1). Las localidades en la Región Andina forman parte de un conjunto de enclaves ubicados en la Cordillera de los Andes, aislados entre sí por ecosistemas húmedos (Soriano \& Ruiz 2003). El Litoral Central y la Costa Oriental conforman el cinturón árido de la Costa CentroOriental, y la Región Noroccidental se ubica en la zona de montañas bajas y depresiones de los Estados Lara y Falcón (Soriano \& Ruiz 2003). De acuerdo con la precipitación, todas estas regiones corresponden a ecosistemas áridos y semiáridos, con promedios anuales que oscilan entre 60 y 600mm (McGuinnies 1979). Los elementos arbóreos dominantes de la vegetación circundante son cactáceas columnares y leguminosas. Las localidaes en la Costa Centro Occidental son afloramientos rocosos, con promedios de precipitaciones anuales superiores a los $600 \mathrm{~mm}$ y la vegetación predominante incluye especies de gramíneas y A. cocui. En el Cuadro 1 se indican datos geográficos y de precipitación de las localidades estudiadas.
Especie de estudio: A. cocui es una agavácea semélpara de distribución amplia en las zonas áridas y semiáridas y afloramientos rocosos de Venezuela y Colombia (García-Mendoza 2004, Albesiano \& Fernández-Alonso 2006). Los especímenes de referencia están depositados en el Herbario Nacional de Venezuela (VEN) (números de catálogo: 213490, 216949, 245367, 347091, 383044). Esta especie es comúnmente conocida como el "cocuy de penca", "maguey" o "cocuiza", y habita en ambientes secos, semiáridos y áridos, asociados con afloramientos rocosos y suelos arenosos. Las hojas son curvadas en la base y verde grisáceas, con espinas de color rojizo en los márgenes y el ápice. Las flores son amarillas, hermafroditas, protándricas y agrupadas en umbelas sobre pedúnculos laterales en un escapo floral que puede llegar a alcanzar entre cuatro y seis metros de altura (Hoyos 1985, Lemus 2003). Además, esta especie presenta autoincompatibilidad genética, lo cual promueve la producción de semillas por vía de la fertilización cruzada (Lemus 2003). La polinización es principalmente llevada a cabo por murciélagos glosofaginos; sin embargo, otros animales como abejas, avispas, mariposas, colibríes y otras aves, también visitan sus flores

CUADRO 1

Localidades escogidas para el estudio de la fenología reproductiva de Agave cocui en Venezuela

TABLE 1

Localities selected to study reproductive phenology of Agave cocui in Venezuela

\begin{tabular}{|c|c|c|c|c|}
\hline Región & Localidad & $\begin{array}{c}\text { PP anual } \\
(\mathrm{mm})\end{array}$ & Coordenadas geográficas & $\begin{array}{l}\text { Altitud } \\
\text { (m.s.n.m) }\end{array}$ \\
\hline \multirow[t]{2}{*}{ Los Andes } & San Antonio (SA), Trujillo & 598.1 & $09^{\circ} 03{ }^{\prime} 25.0^{\prime \prime} \mathrm{N}-070^{\circ} 42^{\prime} 8.1^{\prime \prime} \mathrm{W}$ & 1477 \\
\hline & El Anís (EA), Mérida & 528.6 & $08^{\circ} 27^{\prime} 47.6^{\prime \prime} \mathrm{N}-071^{\circ} 29^{\prime} 32.3^{\prime \prime} \mathrm{W}$ & 516 \\
\hline \multirow[t]{2}{*}{ Noroccidente } & Santa Cruz de Pecaya (SCP), Falcón & 463.5 & $11^{\circ} 05^{\prime} 25.91^{\prime \prime} \mathrm{N}-069^{\circ} 53^{\prime} 00.85^{\prime \prime} \mathrm{W}$ & 237 \\
\hline & San Pablo (SP), Lara & 510.4 & $10^{\circ} 07^{\prime} 52.4^{\prime \prime} \mathrm{N}-069^{\circ} 39^{\prime} 43.0^{\prime \prime} \mathrm{W}$ & 937 \\
\hline \multirow[t]{2}{*}{ Centro Occidente } & La Mona (LM), Carabobo & 1143.2 & $10^{\circ} 06^{\prime} 35.9^{\prime \prime} \mathrm{N}-068^{\circ} 11^{\prime} 09.6^{\prime \prime} \mathrm{W}$ & 765 \\
\hline & La Victoria (LV), Aragua & 987.6 & $10^{\circ} 17^{\prime} 30.8^{\prime \prime} \mathrm{N}-067^{\circ} 19^{\prime} 50.8^{\prime \prime} \mathrm{W}$ & 697 \\
\hline \multirow[t]{2}{*}{ Costa Centro Oriental } & Naiguatá (NA), Vargas & 599.2 & $10^{\circ} 36^{\prime} 47.9^{\prime \prime} \mathrm{N}-066^{\circ} 46^{\prime} 32.6^{\prime \prime} \mathrm{W}$ & 4 \\
\hline & Boca de Uchire (BU), Anzoátegui & 618.2 & $10^{\circ} 05^{\prime} 17.5^{\prime \prime} \mathrm{N}-065^{\circ} 14^{\prime} 32.5^{\prime \prime} \mathrm{W}$ & 10 \\
\hline
\end{tabular}

Entre paréntesis los acrónimos de cada población.

Acronyms of each population are included in parentheses. 
polinizándolas (Lemus 2003). A pesar de que no se han identificado los murciélagos polinizadores, Leptonycteris curasoae y Glossophaga longirostris califican como las dos especies responsables de la polinización de A. cocui, puesto que la distribución de ambas coincide con la de la planta, y además existe evidencia indirecta de este hecho por la presencia de polen de agave sobre el cuerpo de ejemplares de L. curasoae capturados en Falcón y la Costa Oriental de Venezuela (J.M. Nassar \& A. García com. pers.) y también por la presencia de polen en las heces analizadas de esta especie en Lagunillas, Estado Mérida, Venezuela (Sosa \& Soriano 1993). Los frutos son de tipo cápsula, con semillas discales, ásperas y negras dispersadas por el viento. La especie presenta reproducción asexual que ocurre a partir de bulbilos, brotes axilares y basales (Lemus 2003, Infante et al. 2006). Esta planta es empleada en la producción de fibras, medicinas, alimentos y un licor denominado "cocuy de penca", cuya elaboración depende de la extracción de rosetas directamente de las poblaciones silvestres (CETIC-Fundacite-Coro 2007).

\section{Monitorización de fenología reproducti-}

va: En cada una de las localidades de estudio se seleccionó un área con presencia de A. cocui en la que al menos algunos de los individuos presentaran escapo en su fase inicial de emergencia. Como mínimo, en el parche seleccionado debía haber 50 rosetas de dimensiones similares a aquellas que presentaban escapo. El estudio se realizó a lo largo de un año, iniciándose el 26 de septiembre de 2008 y culminando el 15 de septiembre de 2009. Se realizaron visitas a las localidades escogidas cada dos meses, manteniéndose fija el área de registro escogida inicialmente, y se cuantificó la cantidad de individuos presentes en cada una de las siguientes fenofases: escapo emergiendo (individuos con escapo floral en proceso de crecimiento, en los que aún no se observan los primordios florales, Fig. 1-A), flores (individuos con los escapos bien formados con primordios florales y/o las flores ya abiertas, tanto de fase masculina como femenina, Fig. 1B1 y 1B2), frutos inmaduros (escapos con frutos verdes de distintos tamaños, incluso con parte del perianto de las flores ya marchitas, Fig. 1C), frutos maduros (escapos con las cápsulas completamente formadas y de color amarillo, marrón y ya abiertas, Fig. 1D), bulbilos (escapos con bulbilos o propágalos vegetativos de distintos tamaños, Fig. 1E), y escapo seco (son los escapos a los que ya no les queda ninguna de las fenofases anteriores y la parte vegetativa está completamente seca).
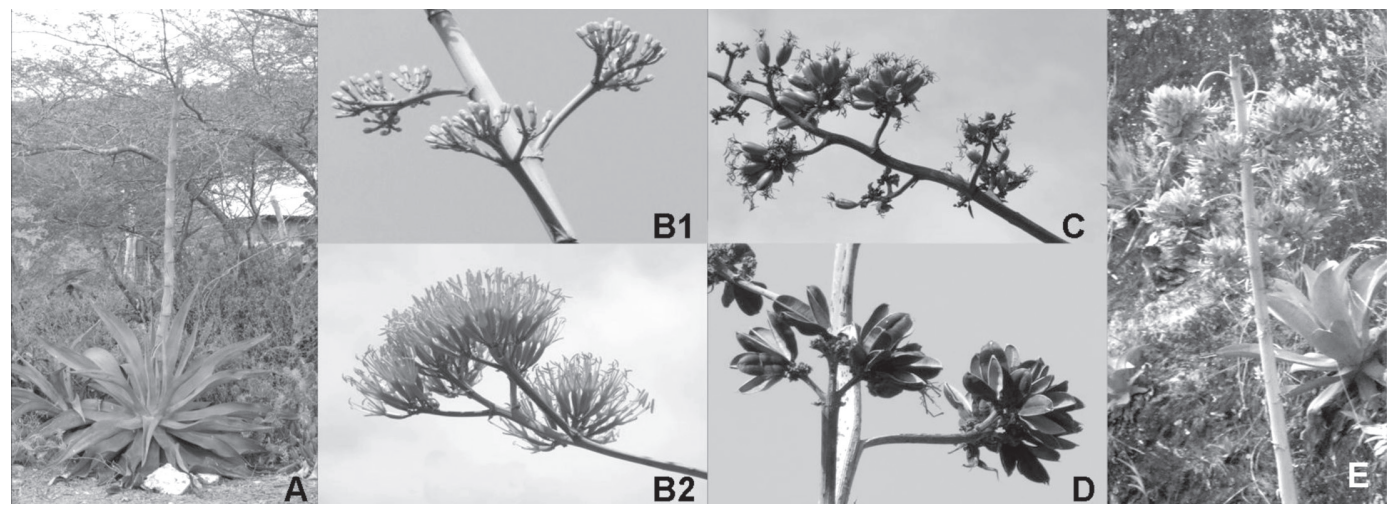

Fig. 1. Estados fenológicos de Agave cocui. (A) Escapo floral, (B1) primordios florales en el escapo floral,(B2) flores abiertas en fase masculina, (C) frutos inmaduros, (D) frutos maduros, cápsulas abiertas y (E) bulbilos. (Fotos: Carmen J. Figueredo). Fig. 1. Phenological stages of Agave cocui: (A) flowering stalk, (B1) inmature flowers in the flowering stalk, (B2) flowers open in the male phase, (C) inmature fruits, (D) mature fruits with open capsules and (E) bulbils. (Photo: Carmen J. Figueredo). 
Para realizar las observaciones de fenología se usaron binoculares, y en el área escogida se tomaron fotos digitales panorámicas de alta resolución con una cámara Fuji 10x, que permitió la complementación de los datos registrados directamente en el campo.

Para cada localidad analizada, se estimó el porcentaje de presencia de individuos en cada fenofase $(P F=$ Número de individuos en fenofase x 100/número total de individuos censados), se generaron fenogramas de líneas múltiples para cada una de las localidades en función del tiempo y se adicionó un segundo eje para indicar el patrón de precipitación promedio histórico de las estaciones climáticas más cercanas a las localidades estudiadas. Para obtener el patrón de precipitación, se tomaron los datos del Ministerio del Ambiente (2010) comprendidos entre 1984 y 1994. Asimismo, se estimaron coeficientes de correlación de rangos de Spearman $(r)$ entre la precipitación promedio mensual y el porcentaje de individuos en floración por localidad para comprobar si existía una asociación significactiva entre ambos parámetros. Con la intención de evaluar la sincronía floral, se determinó el Indice de Solapamiento de Fenofases (ISF) entre localidades. La fórmula para calcular este índice es equivalente a la ecuación del Indice de Solapamiento de Nicho de Pianka (1986) con modificaciones de Nassar \& Emaldi (2008): $I S F=\sum p_{\mathrm{ij}} p_{\mathrm{ik}} / \sum \sqrt{ } p^{2}{ }_{\mathrm{ij}} \sum p_{\mathrm{ik}}^{2}$; donde $p=$ proporción de individuos mostrando una fenofase dada en el episodio $i$ de monitoreo respecto a la suma de individuos mostrando dicha fenofase a través de $n$ monitoreos, con $j$ y $k$ como subscritos del par de poblaciones comparadas. A diferencia del Índice de Solapamiento de Nicho de Pianka, aquí se evalúa la superposición temporal de la floración entre dos poblaciones (p.ejemplo SCP vs. NA) comparando la proporción de individuos en flor para cada período de muestreo dado (septiembre, noviembre, etc.) entre dichas poblaciones. De manera que se generó una matriz pareada de índices de solapamiento de la fenofase floral entre todas las poblaciones examinadas. Al igual que en el Índice de Solapamiento de Nicho de Pianka, un valor igual o cercano a 1 indica un elevado solapamiento de la floración entre las dos poblaciones comparadas; valores cercanos a 0 son indicativos de un bajo grado de solapamiento entre el par de poblaciones.

\section{RESULTADOS}

$\mathrm{Al}$ inicio de la monitorización, en septiembre 2008, todas las localidades con excepación de SCP y NA (ver nombres en el Cuadro 1) exhibieron crecimiento del escapo floral (Fig. 2). Las localidades SP, SA y EA presentaron los máximos de desarrollo de esta estructura entre septiembre y noviembre. Las localidades de la Región Central y Oriental (LM, LV y BU) lo hicieron durante el mes de noviembre. La población de SA fue la primera en iniciar la floración en el mes de septiembre, seguida de LM en noviembre. Para las localidades restantes la floración se inició en enero. Los máximos de floración correspondieron con la época de sequía, que se extiende desde enero hasta marzo en todas las localidades estudiadas. En la Fig. 2 se observa que la mayor producción de primordios florales y flores ocurrió en los meses de menor precipitación del promedio histórico para las ocho localidades. Conjuntamente, la correlación entre la precipitación promedio mensual y la producción de flores en todas las localidades fue negativa y significativa en varias poblaciones (ver valores de $r$ en Fig. 2), lo que indica que a medida que disminuyen las lluvias hay un aumento de la producción de flores. Las localidades de SP y LM fueron las que exhibieron el intervalo más corto de floración, de unos cuatro meses aproximadamente. Para el resto de las poblaciones esta fenofase duró entre cinco y seis meses. Los máximos de producción de frutos inmaduros se presentaron entre enero y mayo en función de la población analizada (enero: SA; marzo: SP, LM y LV; mayo: EA, SCP, NA y BU; Fig. 2). Mientras que con relación a los frutos maduros, los máximos de producción ocurrieron entre marzo y mayo (marzo: SCP, EA, LM; mayo: SP, SA, LV, NA y BU; Fig. 2).

En cuanto a la reproducción asexual, la producción de bulbilos se inicia durante la 

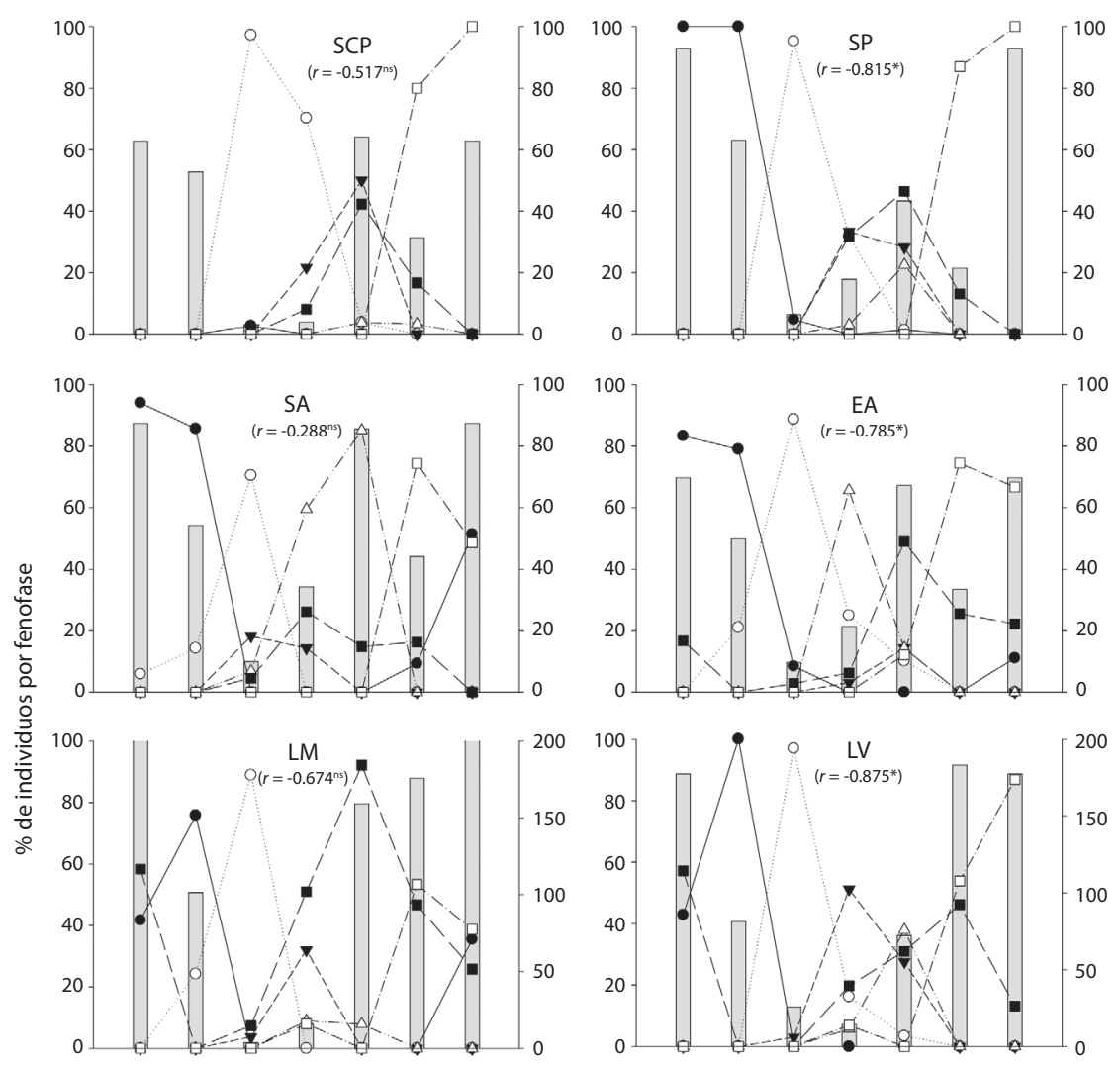

100
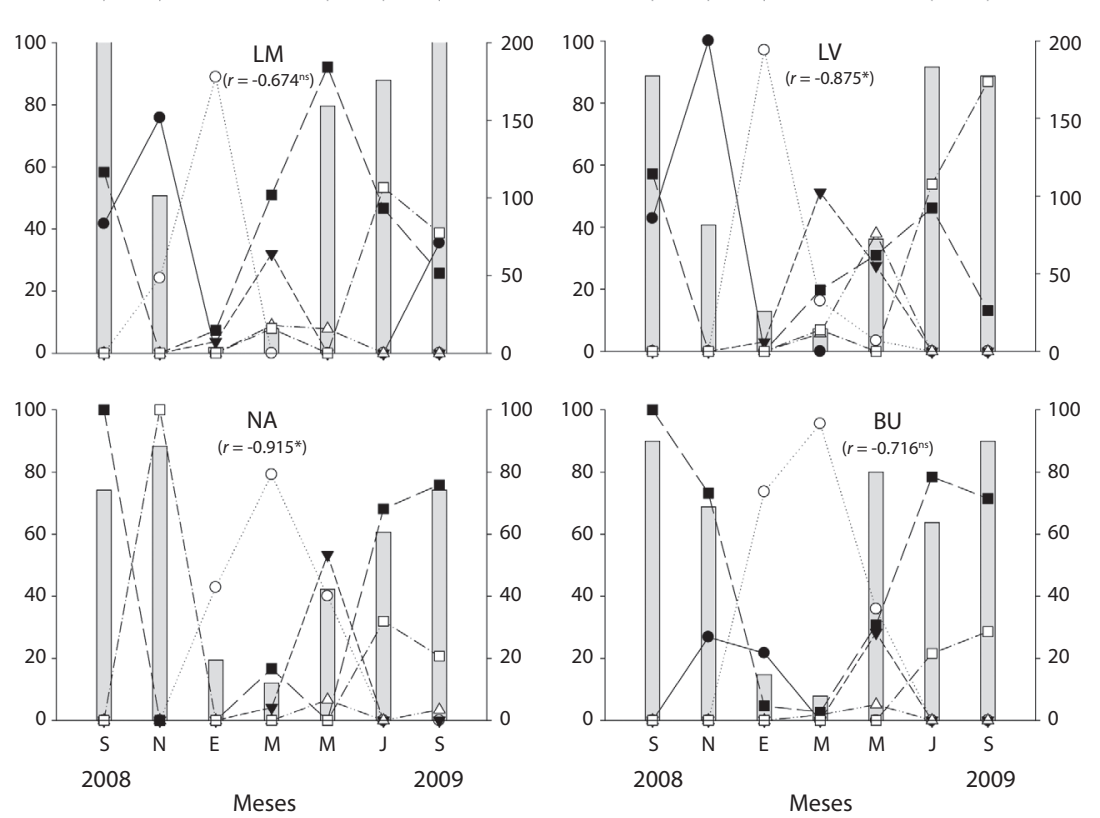

Fig. 2. Fenogramas reproductivos de las poblaciones de Agave cocui en las regiones Noroccidental, Andina, Centro Occidental, Costa Centro Oriental de Venezuela $(\bullet=$ =scapo saliendo; $O=$ primordios y flores; $\boldsymbol{\nabla}=$ frutos verdes; $\triangle=$ frutos maduros; $\boldsymbol{\square}=$ bulbilos; $\square$ =escapos secos, barras grises=precipitación). En barras grises se muestran los patrones de precipitación promedio históricos desde 1984 hasta 1994 (Datos de precipitación Minamb 2010) SCP=Santa Cruz de Pecaya, SP=San Pablo, SA=San Antonio, EA=El Anís, LM=La Mona, LV=La Victoria, NA=Naiguatá, BU=Boca de Uchire. Se indican valores de coeficientes de correlación de Spearman, ns: correlación no significativa, *correlación significativa con $\mathrm{p}<0.05$.

Fig. 2. Phenograms reproductive Agave cocui populations in the Northwest region, Andean, Central Western and Central Eastern Coast of Venezuela $(\boldsymbol{\bullet}=$ stalks out; $\mathrm{O}=$ =inmature and mature flowers, $\boldsymbol{\nabla}$ =inmature fruit, $\triangle=$ mature fruit, $\boldsymbol{\nabla}=$ bulbils; $\square=$ dry stalks, gray bars=precipitation). Gray bars show the average historical rainfall patterns from 1984 to 1994 (Data of precipitation Minamb 2010). SCP=Santa Cruz de Pecaya, SP=San Pablo, SA=San Antonio, EA=El Anís, LM=La Mona, LV=La Victoria, NA=Naiguatá, BU=Boca de Uchire. The values indicate Spearman correlation coefficient, ns: correlation not significant, *significant correlation with $\mathrm{p}<0.05$. 
época de sequía, pero los máximos de producción de estas estructuras se ubicaron durante los meses con presencia de lluvias para todas las localidades. En el campo se tuvo la oportunidad de observar individuos que presentaron todo el escapo cubierto con cápsulas o con bulbilos. Estas condiciones pudieran sugerir la efectividad o no del proceso de polinización. A medida que transcurrieron los meses, los bulbilos fueron cayendo al suelo, hasta que el escapo se secó completamente. La localidad de LM exhibió una proporción relativamente elevada de individuos con bulbilos (>90\%).

En cuanto al grado de sincronía del proceso de floración, las poblaciones analizadas se pueden separar en dos grupos de acuerdo a los índices de solapamiento de esta fenofase (Cuadro 2); un primer grupo con elevada sincronía floral (ISF=0.755 y 0.999), conformado por SCP, SP, SA, EA, LM y LV, que abarca la Región Andina, Noroccidente y Centro. El segundo grupo consta de las dos poblaciones ubicadas en la Costa Central y Oriental del país, NA y BU, también con elevada sincronía floral $(I S F=0.971)$. El máximo de floración de este grupo ocurrió en marzo, mostrando así un retraso de dos meses con respecto al primer grupo de poblaciones (Fig. 2). Este desfase temporal entre los dos grupos se ve reflejado en los valores más bajos de $I S F$ registrados entre algunos de los pares de poblaciones conformados por representantes de ambos grupos (0.286 - 0.658, Cuadro 2). Sin embargo, cabe señalar que las comparaciones pareadas de las localidades de BU y NA con SCP mostraron valores altos de $I S F$ (0.907 y 0.784 , respectivamente), debido a que la población de SCP mantuvo una proporción elevada de individuos con yemas florales y con flor hasta marzo, lo cual coincide con los máximos de floración. De igual forma ocurrió con la población de BU, en la que una proporción alta de individuos en flor ocurrió durante los máximos de floración de SCP y SP.

\section{DISCUSIÓN}

Agave cocui presenta un elevado grado de sincronía reproductiva en su área de distribución en Venezuela, siendo la época de sequía la ventana de tiempo en la cual se concentra el período de floración de esta especie. Este solapamiento reproductivo es particularmente pronunciado en la región Centro Occcidental del país, desde la costa hasta los Andes. Esta condición pudiera facilitar el flujo génico interpoblacional vía polen a lo largo de ese eje

\section{CUADRO 2}

Matriz pareada del Índice de Solapamiento de Fenofases (ISF) entre las ocho localidades estudiadas de Agave cocui en Venezuela

TABLE 2

Matrix of pairwise Phenophase Overlapping Index (POI) among the eight localities where Agave cocui was studied in Venezuela

\begin{tabular}{|c|c|c|c|c|c|c|c|}
\hline Localidad & $\begin{array}{c}\text { Santa Cruz } \\
\text { de Pecaya } \\
\text { (SCP) }\end{array}$ & $\begin{array}{l}\text { San Pablo } \\
\text { (SP) }\end{array}$ & $\begin{array}{c}\text { San Antonio } \\
\text { (SA) }\end{array}$ & $\begin{array}{c}\text { El Anís } \\
\text { (EA) }\end{array}$ & $\begin{array}{c}\text { La } \\
\text { Mona } \\
\text { (LM) }\end{array}$ & $\begin{array}{c}\text { La } \\
\text { Victoria } \\
\text { (LV) }\end{array}$ & $\begin{array}{c}\text { Naiguatá } \\
\text { (NA) }\end{array}$ \\
\hline Santa Cruz de Pecaya (SCP) & - & & & & & & \\
\hline San Pablo (SP) & 0.927 & - & & & & & \\
\hline San Antonio (SA) & 0.755 & 0.943 & - & & & & \\
\hline El Anís (EA) & 0.852 & 0.978 & 0.979 & - & & & \\
\hline La Mona (LM) & 0.756 & 0.943 & 0.999 & 0.979 & - & & \\
\hline La Victoria (LV) & 0.909 & 0.999 & 0.955 & 0.986 & 0.955 & - & \\
\hline Naiguatá (NA) & 0.784 & 0.546 & 0.286 & 0.473 & 0.286 & 0.525 & - \\
\hline Boca de Uchire (BU) & 0.907 & 0.728 & 0.495 & 0.658 & 0.496 & 0.709 & 0.971 \\
\hline
\end{tabular}


geográfico, además de promover el establecimiento de un corredor de néctar y polen para especies dependientes de estos recursos nutricionales en las zonas áridas y semiáridas en el Occidente de Venezuela.

La asociación de la actividad reproductiva de $A$. cocui con la época seca sugiere que la estrategia reproductiva de esta suculenta corresponde con la de plantas árido-tolerantes (Fischer \& Turner 1978, Pavón \& Briones 2001), especies en las que distintas funciones biológicas dependen de las reservas de agua acumuladas en la propia planta. Por su parte, la dehiscencia de los frutos en el momento en que hay un aumento en las precipitaciones, permite que las semillas dispongan de agua en el suelo para germinar y establecerse durante el período de lluvias. En general, la concentración de la floración durante la estación seca ha sido reportada para otras especies del género Agave, incluyendo A. macroacantha Zucc., A. salmiana Otto ex Salm-Dyck, A. lechuguilla Torr., A. schotti Engelm., A. difformis A. Berger, A. garcia-mendozae Galvan \& L. Hern. y A. striata Zucc., todas distribuidas en México (Martínez del Río \& Eguiarte 1994, Trame et al. 1995, Arizaga et al. 2000ab, Arizaga \& Ezcurra 2002, Molina-Freaner \& Eguiarte 2003, SilvaMontelllano \& Eguiarte 2003, Rocha 2006), lo que sugiere que ésta estrategia reproductiva está ampliamente extendida dentro de este género de plantas.

La sincronización del proceso de floración en $A$. cocui a escala regional podría permitir el intercambio de genes vía polen entre poblaciones en parte del área de distribución de esta especie en Venezuela. Para que el flujo génico mediado por polen ocurra sobre largas distancias, es necesario que la viabilidad del polen se mantenga al menos por el tiempo necesario para que éste sea transportado entre poblaciones. En este sentido, para A. habardiana Trelease se reporta que los granos de polen permanecen viables por al menos tres días (Kuban 1989). Por otra parte, también es necesario que el agente polinizador sea capaz de transportar polen largas distancias en un tiempo relativamente corto. El murciélago de los cardones,
Leptonycteris curasoae, tiene capacidad de vuelo sobre largas distancias, y se ha propuesto que posiblemente realiza movimientos migratorios anuales en el Occidente del país (Soriano et al. 2000, Fleming \& Nassar 2002, Newton et al. 2003). Por lo tanto, si A. cocui exhibe un período de viabilidad de polen similar al de $A$. habardiana y efectivamente $L$. curasoae realiza movimientos migratorios a lo largo de eje árido Norte-Sur en el Occidente de Venezuela, entonces es posible que este polinizador utilice las poblaciones de A. cocui en flor como un corredor de néctar y polen durante los primeros meses del año, contribuyendo así con la generación de eventos de flujo genético de larga distancia entre las poblaciones de esta especie. Es precisamente entre diciembre y abril cuando L. curasoae desaparece de las cuevas donde se refugia en la Península de Paraguaná, en el Norte del Estado Falcón (Soriano et al. 2000, J.M. Nassar, obs. pers.). Por otra parte, el desfase detectado en la floración entre las poblaciones del Litoral Central y Oriente del país y la Región Noroccidental, hacen menos factible que ocurran eventos de flujo genético entre ambas regiones.

La producción de bulbilos en esta especie fue alta en algunas poblaciones y se inició inmediatamente después de la producción de las flores. Por lo tanto, se ha encontrado que en agaves los bulbilos se generan a partir de meristemas no diferenciados en los pedicelos de las flores, cuando éstas abortan (Gentry 1982, Hodgson et al. 1989, Arizaga \& Ezcurra 1995, Nobel 2003). A. cocui no exhibe viviparía, lo que ocurre es la formación de bulbilos a expensas de las estructuras sexuales (GómezPompa 1963, Bell 1991). Los meristemas que dan origen a los bulbilos son grupos de células no diferenciadas derivadas por mitosis que no experimentan meiosis, y por tanto esta condición debería generar baja variabilidad genética en las poblaciones donde predomina la reproducción clonal (Infante et al. 2003).

Las flores de A. cocui no llegan a formar frutos por dos posibles razones. Es probable que ocurran abortos pre o postfecundación si se dan eventos de autopolinización, puesto 
que esta especie es autoincompatible (Lemus 2003). Aunque dentro de una umbela las flores presentan la fase masculina o femenina, dentro de un mismo escapo en flor distintas ramas pueden estar en una u otra fase, haciendo posible que ocurran eventos de geitonogamia. Por otro lado, puede ocurrir limitación por polinizadores. Aunque la distribución geográfica de A. cocui en la región Noroccidental y los Andes coincide con la distribución de L. curasoae y G. longirostris (Nassar et al. 2003), en otras localidades con presencia de A. cocui, como es el caso del afloramiento rocoso de la población de La Mona (LM), la presencia de estos murciélagos no está reportada, y por tanto es probable que exista un déficit de polinizadores para esta planta. De hecho, esta población fue la que presentó uno de los porcentajes más elevados de presencia de bulbilos (>90\%). Adicionalmente, puede que los primordios florales, las flores abiertas y las flores ya polinizadas estén siendo depredadas. En este sentido, se ha registrado daño en las flores de $A$. cocui por larvas de Phidotricha erigen Ragonot (Lepidoptera: Pyralidae), así como por el picudo del agave, Scyphophorus acupunctatus Gyllenhaal (Velásquez et al. 2007), los cuales estarían contribuyendo al aborto de las flores.

En resumen, A. cocui es una especie con marcada estacionalidad reproductiva en ambientes áridos y semiáridos de Venezuela, con un período de floración principalmente ubicado a lo largo de la estación seca. La elevada sincronía reproductiva de esta especie a lo largo del eje Norte-Sur en el Occidente del país, hace posible la existencia de un corredor de néctar y polen para aves y murciélagos nectarívoros-polinívoros que dependen de los recursos florales de esta planta. Es posible que algunos de estos visitantes florales pudieran estar moviéndose a lo largo de dicho corredor, propiciando el flujo genético vía polen entre poblaciones de A. cocui. Pero la permanencia de este hipotético corredor de néctar y el intercambio genético entre poblaciones de agave pudieran estar bajo amenaza, ya que esta planta es objeto de una creciente presión de uso para la producción de bebidas espirituosas en la región Noroccidental (Savedra et al. 2006, CETIC-Fundacite-Coro 2007, Padilla et al. 2007), actividad que sin control podría concluir con la extinción de algunas de las poblaciones que conforman el corredor de agaves que se extiende desde Venezuela hasta Colombia.

\section{AGRADECIMIENTOS}

Los autores agradecen al Instituto Venezolano de Investigaciones Científicas (IVIC) y al Plan de Desarrollo de Talento Humano de Alto Nivel del Fondo Nacional de Ciencia y Tecnología (FONACIT) (Proyecto S1-2002000358) por el apoyo financiero para la realización de este proyecto. Gonzalo Medina y Marianne Asmüssen asistieron en la toma de datos en el campo. Tres revisores anónimos de este manuscrito aportaron valiosos comentarios y sugerencias para el mejoramiento del mismo.

\section{RESUMEN}

Agave cocui (Agavaceae) es una especie de amplia distribución en zonas áridas y semiáridas de Venezuela y Colombia. A pesar de su importancia ecológica como fuente de recursos para la fauna silvestre y de su potencial valor económico como materia prima para la producción del licor "Cocuy de Penca", los estudios sobre su ecología reproductiva son muy escasos. En este estudio se evaluó la fenología de floración y fructificación de A. cocui en ocho localidades de Venezuela. La fase reproductiva se inició al finalizar el periodo de lluvias. Las flores se producen durante cinco meses. Para la Región Noroccidental del país y los Andes los máximos de floración ocurrieron en enero, mientras que las localidades en la costa central y oriental exhibieron un desface de cerca de dos meses, a principios de marzo. La mayor sincronía floral ocurrió entre todas las poblaciones censadas en el occidente del país, incluyendo las localidades andinas. Dicha sincronía reproductiva potenciaría la conformación de un corredor de néctar para aves y murciélagos, que se extendería desde el Norte de Falcón, en la Costa Occidental, hasta los bolsones andinos, en el Suroeste del país.

Palabras clave: Agavaceae, corredor de néctar, floración, sincronía reproductiva, zonas áridas. 


\section{REFERENCIAS}

Albesiano, S. \& J.L. Fernández-Alonso. 2006. Catálogo comentado de la flora vascular de la franja tropical (500-1200 m) del Cañón del Río Chicamocha (Boyacá-Santander, Colombia). Primera Parte. Caldasia 28: 23-44.

Arizaga, S. \& E. Ezcurra. 1995. Insurance against reproductive failure in a semelparous plant: bulbil formation in Agave macroacantha flowering stalks. Oecologia 101: 329-334.

Arizaga, S. \& E. Ezcurra. 2002. Propagation mechanisms in Agave macroacantha (Agavaceae), a tropical aridland succulent rosette. Am. J. Bot. 89: 632-641.

Arizaga, S., E. Ezcurra, A.F. Ramírez de Arellano \& E. Vega. 2000a. Pollination ecology of Agave macroacantha (Agavaceae) in a Mexican tropical desert. II. The role of pollinators. Am. J. Bot. 87: 1011-1017.

Arizaga, S., E. Ezcurra, E. Peters, A.F. Ramírez de Arellano \& E. Vega. 2000b. Pollination ecology of Agave macroacantha (Agavaceae) in a Mexican tropical desert. I. Floral biology and pollination mechanisms. Am. J. Bot. 87: 1004-1010.

Bell, A.D. 1991. Plant form. An illustrated guide to flowering plant morphology. Oxford University, Londres, Inglaterra.

Borchert, R., S.A. Meyer, R.S. Felger, L. Porter-Bolland. 2004. Environmental control of lowering periodicity in Costa Rican and Mexican tropical dry forests. Global Ecol. Biogeogr. 13: 409-425.

Brusca, R.C., T.A. Hubbard, T.R. Van Devender, M.A. Dimmitt, K. Krebbs, Y. Gray, T.H. Fleming \& C. Martínez del Río. 2009. Arizona-Sonora Desert Museum. (Consultado: 20 de octubre 2009, www. desertmuseum.org.htm).

Ceballos, G., T.H. Fleming, C. Chávez, \& J.M. Nassar. 1997. Population dynamics of Leptonycteris curasoae (Chiroptera: Phyllostomidae) in Jalisco, Mexico. J. Mammal. 78: 1220-1230.

CETIC-Fundacite-Coro. 2007. Red de innovación productiva del Agave cocui (Consultado: 10 de octubre 2009, http://cetic.fundacite-falcon.gob.ve/ced/ cocuy/c1/).

Eamus, D. \& L. Prior. 2001. Ecophysiology of trees of seasonally dry tropics: Comparisons among phenologies. Adv. Ecol. Res. 32: 113-197.
Elliott, S, P.J. Baker \& R. Borchert. 2006. Leaf-flushing during the dry season: The paradox of Asian monsoon forests. Global Ecol. Biogeogr. 15: 248-257.

Fischer, R.A. \& N.C. Turner. 1978. Plant productivity in the arid and semiarid zones. Annu. Rev. Plant. Physiol. 29: 277-317.

Fitter, A.H \& R.S.R. Fitter. 2002. Rapid change in flowering time in British plants. Science 296: 1689-1692.

Fleming, T.H., R.A. Nuñez \& L.S. Sternberg. 1993. Seasonal-Changes in the Diets of Migrant and Non-Migrant Nectarivorous Bats as Revealed by Carbon Stable Isotope Analysis. Oecologia 94: 72-75.

Fleming, T.H. \& J.M. Nassar. 2002. Population biology of the Lesser Long-nosed bat Leptonycteris curasoae in Mexico and Northern South America, p. 283-305. In T.H. Fleming \& A. Valiente-Banuet (eds.). Columnar cacti and their mutualists: evolution, ecology, and conservation, University of Arizona, Tucson, Arizona, EEUU.

Fresnillo-Fedorenko, D.E., O.A. Fernández, C.A. Busso \& O.E. Elõa. 1996. Phenology of Medicago minima and Erodium cicutarium in semiarid Argentina. J. Arid Environ. 33: 409-416.

García-Mendoza, A. 2004. Agavaceae, p. 673. In O. Hokche, P.E. Berry \& O. Huber (eds.). Nuevo catálogo de la flora vascular de Venezuela. Fundación Instituto Botánico de Venezuela, Caracas, Venezuela.

Gentry, H.S. 1982. Agaves of continental North America. University of Arizona, Tucson, Arizona, EEUU.

Gómez-Pompa, A. 1963. El género Agave. Cact. Suc. Mex. 8: $3-25$.

Hodgson, W.C., G.P. Nabhan \& L. Ecker. 1989. Conserving rediscovered Agave cultivars. Agave 3: 9-11.

Hoyos, J. 1985. Flora de la Isla de Margarita. Sociedad Fundación La Salle de Ciencias Naturales, Caracas, Venezuela.

Infante, D., S. Molina, J.R. Demey \& E. Gamez. 2006. Asexual genetic variability in Agavaceae determined with inverse sequence-tagged repeats and amplification fragment length polymorphism analysis. Plant Mol. Biol. Rep. 24: 205-217.

Janzen, D.H. 1967. Synchronization of sexual reproduction of trees within the dry season in Central America. Evolution 21: 620-37.

Kuban, J.F. 1989. The pollination biology of two populations of the Big Bend century plant, Agave 
havardiana Trel.: a multiple pollinator syndrome with floral specialization for vertebrate pollinators. Tesis de Doctorado, Syracuse University, Syracuse, Nueva York, EEUU.

Lemus, L. 2003. Ecología reproductiva de Agave cocui Trelease (Agavaceae) en zonas áridas del estado Falcón, Venezuela: I. fenología reproductiva, biología floral, mecanismo de polinización y sistema genético de reproducción. Croizatia 4: 36-48.

Martínez del Río, C. \& L.E. Eguiarte. 1994. Bird visitation to Agave salmiana: comparison among hummingbirds and perching birds. Condor 89: 357-363.

McGinnies, W.G. 1979. General description of desert areas, p. 5-19. In Goodall, D \& R. Perry (eds.) Arid land ecosystems: structure, functioning, and management. Cambridge University, Cambridge, EEUU.

Ministerio del Poder Popular para el Ambiente. 2010. Agua, Precipitación Mensual (Consultado: 17 de marzo 2010, www.minamb.gob.ve.htm).

Molina-Freaner, F. \& L.E. Eguiarte. 2003. The pollination biology of two paniculate Agaves (Agavaceae) from northwestern Mexico: Contrasting roles of bats as pollinators. Am. J. Bot. 90: 1016-1024.

Mosquin, T. 1971. Competition for pollinators as a stimulus for the evolution of flowering time. Oikos 22: 398-402.

Nassar, J.M. \& U. Emaldi. 2008. Fenología reproductiva y capacidad de regeneración de dos cardones, Stenocereus griseus (Haw.) Buxb. y Cereus repandus (L.) Mill. (Cactaceae). Acta Bot. Venez. 31: 495-528.

Nassar, J.M., H. Beck, L.S.L. Sternberg \& T.H. Fleming. 2003. Dependence on cacti and Agaves in nectarfeeding bats from Venezuelan arid zones. J. Mammal. 84: 106-116.

Newton, L.R., J.M. Nassar \& T.H. Fleming. 2003. Genetic population structure and mobility of two nectarfeeding bats from Venezuelan deserts: inferences from mitochondrial DNA. Mol. Ecol. 12: 3191-3198.

Nobel, P.S. 2003. Environmental biology of Agaves and Cacti. Cambridge University, Cambridge, EEUU.

Noma, N. \& T. Yumoto. 1997. Fruiting phenology of animal-dispersed plants in response to winter migration of frugivores in a warm temperate forest on Yakushima Island, Japan. Ecol. Res. 12: 119-129.

Padilla, A., S. Savedra \& D. Padilla. 2007. Impacto al ecosistema semiárido atribuido a la elaboración de cocuy pecayero en Falcón, Venezuela. Rev. Fac. Agron. Univ. Zulia 24: 158-163.

Pavón, N.P. \& O. Briones. 2001. Phenological patterns of nine perennial plants in an intertropical semi-arid Mexican scrub. J. Arid. Environ. 49: 265-277.

Pianka, E.R. 1986. Ecology and natural history of desert lizards. Princeton University, New Jersey, EEUU.

Rivera, G., S. Elliott, L.S. Caldas, G. Nicolossi, V.T.R. Coradin \& R. Brochert. 2002. Increasing day-length induces spring flushing of tropical dry forest trees in the absence of rain. Trees 16: 445-456.

Rocha, M., S.V. Good-Avila, F. Molina-Freaner, H.T. Arita, A. Castillo, A. García-Mendoza, A. SilvaMontellano, B.S. Gaut, V. Souza \& L.E. Eguiarte. 2006. Pollination biology and adaptive radiation of Agavaceae, with special emphasis on the genus Agave. Aliso 22: 329-344.

Savedra, S., A. Padilla \& D. Padilla. 2006. Especies forestales usadas en la producción de cocuy pecayero en el Estado Falcón, Venezuela. Rev. Forest. Latinoamericana 40:71-84.

Silva-Montellano, A. \& L.E. Eguiarte. 2003. Geographic patterns in the reproductive ecology of Agave lechuguilla (Agavaceae) in the chihuahuan desert. II Genetic Variation, differentiation and inbreeding estimate. Am. J. Bot. 90: 700-706.

Soriano, P.J. \& A. Ruiz. 2003. Arbustales xerofíticos, p. 696-715. In M. Aguilera, A. Azócar \& E. GonzálezGiménez (eds.). Biodiversidad en Venezuela. Fundación Polar, Ministerio de Ciencia y Tecnología, Fondo Nacional de Ciencia y Tecnología (FONACIT), Caracas Venezuela.

Soriano, P.J., A. Ruiz \& J.M. Nassar. 2000. Notes on the distribution and ecological importance of the bats Leptonycteris curasoae and Glossophaga longirostris in Andean arid zones. Ecotropicos 13: 91-95.

Sosa, M. \& P.J. Soriano. 1993. Solapamiento de dieta entre Leptonycteris curasoae y Glossophaga longirostris (Mammalia: Chiroptera). Rev. Biol. Trop. 41: 529-532. 
Trame, A.M., A.J. Coddington \& K.N. Paige. 1995. Field and genetic-studies testing optimal outcrossing in Agave schottii, a long-lived clonal plant. Oecologia 104: 93-100.

Velásquez, J., Y. Romero, M. González, M. Meléndez, N. Salas \& Z. Medina. 2007. Principales enfermedades infecciosas del Cocuy (Agave cocui Trelease) en el Estado Falcón - Venezuela. VII Jornadas de Investigación en el marco del 30 aniversario de la Universidad Nacional Experimental Francisco de Miranda (UNEFM).
Waser, N.M. 1979. Pollinator availability as a determinant of flowering time in Ocotillo (Fouquieria splendens). Oecologia 39: 107-21.

Waser, N.M. \& L.A. Real. 1979. Effective mutualism between sequentially flowering plant species. Nature 281: 670-72.

Zeng, Z.G., P.S.A. Beck, T.J. Wang, A.K. Skidmore, Y.L. Song, H.S. Gong \& H.H.T. Prins. 2010. Effects of plant phenology and solar radiation on seasonal movement of golden takin in the Qinling Mountains, China. J. Mammal. 91: 92-100. 